\title{
Gallbladder Torsion Masking as Acalculus Cholecystitis: A Review of Two Cases Including Unsuccessful Management With Percutaneous Cholecystostomy
}

\author{
Jack Cecire $^{\mathrm{a}, \mathrm{b}}$, Andrew Sutherland ${ }^{\mathrm{a}}$, Kamala Kanta Das ${ }^{\mathrm{a}}$
}

\begin{abstract}
Gallbladder torsion is a rare cause of abdominal pain; however, it is important to diagnose pre-operatively. We report two cases of gallbladder torsion, both of which were not recognized until the time of surgery. Both patients were elderly thin females, presenting with right upper quadrant pain, and on examination had a positive Murphy's sign, and pre-operative imaging was suggestive of acalculus cholecystitis. One patient was initially managed by percutaneous insertion of a cholecystostomy tube, with progression to a laparoscopic cholecystectomy after a failure of resolution of symptoms. The second patient was taken to theater shortly after presentation, with the gallbladder torsion recognized intra-operatively. In both cases, the gallbladder was distorted and a routine laparoscopic cholecystectomy was performed with good recovery following. These two cases highlight the need for gallbladder torsion to be considered as a diagnostic possibility in those presenting with right upper quadrant pain, particularly those groups at most risk, including elderly thin females. Whilst other causes of cholecystitis can be managed non-operatively, at least in the acute phase, gallbladder torsion requires urgent operative intervention.
\end{abstract}

Keywords: Gallbladder; Torsion; Volvulus; Cholecystitis; Acute; Acalculus

\section{Introduction}

Gallbladder torsion is rare and occurs secondary to the twisting of the gallbladder on its mesentery. Less than 350 cases of gallbladder torsion have been reported, with the diagnosis of many of these cases only occurring at the time of operation [1]. Anatomical variation is thought to play a role in its pathogenesis, with an abnormally long mesentery joining the

Manuscript submitted February 15, 2021, accepted March 2, 2021

Published online March 20, 2021

${ }^{a}$ Department of Surgery, Coffs Harbour Health Campus, Coffs Harbour, NSW, Australia

${ }^{\mathrm{b}}$ Corresponding Author: Jack Cecire, Department of Surgery, Coffs Harbour Health Campus, Coffs Harbour, NSW, Australia. Email: j.cecire@gmail.com

doi: https://doi.org/10.14740/jmc3683 gallbladder to the liver, resulting in a free-hanging or "floating gallbladder", which was first described by Wendel in 1898 [2]. This gives the gallbladder the mobility required to twist, resulting in torsion [3]. It is theorized that loss of visceral fat and associated shrinkage of the liver result in a gallbladder that hangs free by its mesentery [4]. It is then not suprising that gallbladder torsion has been most frequently observed in elderly thin females [1].

Whilst the vast majority of cases of gallbladder torsion are diagnosed intra-operatively, there are a small number of case reports which report on pre-operative diagnosis via the use of magnetic resonance cholangiopancreatography and computed tomography $(\mathrm{CT})[5,6]$.

Whilst other causes of cholecystitis can be treated nonoperatively, gallbladder torsion requires urgent surgical intervention, because of the risk of ischemia and gangrene leading to perforation, and as such there is a need to increase its preoperative diagnosis.

\section{Case Reports}

\section{Case 1}

An 84-year-old female presented to the emergency department with a 2-day history of right upper quadrant abdominal pain. She had a background of severe chronic obstructive pulmonary disease. Her abdominal examination revealed a tender right upper quadrant with a positive Murphy's sign. Her laboratory results were largely unremarkable with a non-elevated white cell count, a normal bilirubin and liver function tests, excepting a mildly elevated gamma-glutamyl transpeptidase (GGT) and aspartate aminotransferase (AST) $(60 \mathrm{U} / \mathrm{L}$ (reference (ref) $5-50 \mathrm{U} / \mathrm{L}$ ) and $133 \mathrm{U} / \mathrm{L}($ ref $30-110 \mathrm{U} / \mathrm{L})$ ). An abdominal $\mathrm{CT}$ showed a markedly distended gallbladder extending to the right iliac fossa and mild thickening of the gallbladder wall. An ultrasound revealed gallbladder distension with heterogeneous gallbladder wall thickening up to $15 \mathrm{~mm}$ without evidence of gallstones. A diagnosis of acalculous cholecystitis was made, and the patient proceeded to have percutaneous cholecystostomy in light of the gallbladder distension and the patient's comorbidities. The patient failed to improve symptomatically and 8 days later underwent laparoscopy which identified a torsion of the gallbladder. She underwent a laparoscopic chol- 
ecystectomy and recovered well and was discharged on day 4 post-operatively.

\section{Case 2}

The second case is an 81-year-old female, who presented to the emergency department after a 1-day history of right upper quadrant pain. She also had right upper quadrant tenderness with a positive Murphy's sign. She had a markedly elevated neutrophilia at $26.5 \times 10^{9} / \mathrm{L}$. Her liver function tests were largely unremarkable (bilirubin $12 \mu \mathrm{mol} / \mathrm{L}$ (normal $<20$ $\mu \mathrm{mol} / \mathrm{L}$ ), GGT $93 \mathrm{U} / \mathrm{L}$ (ref 5 - $50 \mathrm{U} / \mathrm{L}$ ), alkaline phosphatase (ALP) $105 \mathrm{U} / \mathrm{L}$ (ref $30-110 \mathrm{U} / \mathrm{L}$ ), alamine aminotransferase (ALT) $30 \mathrm{U} / \mathrm{L}$ (ref 10 - $50 \mathrm{U} / \mathrm{L}$ ) and AST $37 \mathrm{U} / \mathrm{L}$ (ref 10 - 35 U/L)). An ultrasound revealed a thickened gallbladder wall up to $9 \mathrm{~mm}$, direct tenderness with probe pressure and no evidence of gallstones. The patient was diagnosed with acalculous cholecystitis. This patient was taken to theater for laparoscopy with intra-operative findings of a gallbladder torsion. The gallbladder was dark and congested without evidence of perforation. The gallbladder was distorted and routine laparoscopic cholecystectomy was performed. Histopathological examination of the gallbladder showed a necrotic wall and features suggestive of acute-on-chronic cholecystitis. She recovered well and was discharged on day 3 after the procedure.

\section{Discussion}

The most common cause of cholecystitis is gallstone disease, caused by transient or permanent obstruction of the cystic duct [7]. Acalculous cholecystitis on the other hand is most often seen in critically ill patients in the intensive care unit and represents somewhere in the vicinity of $2-15 \%$ of cases of cholecystitis $[8,9]$. Gallbladder torsion is a separate entity which can mimic other causes of cholecystitis. Pre-operative diagnosis is important as it requires urgent surgical management. The potential consequence of delaying intervention is ischemia with necrosis and perforation.

Whilst early cholecystectomy is recommended for the treatment of acute calculous cholecsytitis, initial non-operative treatment with delayed cholecystectomy has an adequate success rate, with this path more appropriate for some patient populations, dependent on their risk profile [10-12]. In addition, acalculous cholecystitis is often managed non-operatively with a growing trend towards percutaneous cholecystostomy as the initial and sometimes definitive management $[13,14]$. In contrast, nonoperative management with intravenous antibiotics and percutaneous cholecystostomy, as evidenced by this case study, are not viable management options in the treatment of gallbladder torsion, as it is not primarily an infective process and a percutaneous cholecystostomy does not correct the underlying pathology.

Pre-operative diagnosis remains difficult with presenting symptomatology and pre-operative investigation is similar to alternative causes of cholecystitis.

One case report reports a magnetic resonance imaging (MRI) showing an abnormal gallbladder position, with the fundus lying inferomedial to Hartmann's pouch; however, this finding is not elsewhere reported in the literature [15]. Other studies report a "cystic duct knot sign" on ultrasonography and a CT finding of a "whirl sign" in cases of reported cases of gallbladder torsion $[16,17]$.

Whilst causes of cholecystitis can be managed non-operatively, gallbladder torsion requires emergency surgery and as such requires prompt recognition. Perforation and peritonitis secondary to biliary contamination are known as complications of gallbladder torsion.

\section{Conclusion}

In conclusion, gallbladder torsion should be considered in all cases of acalculus cholecystitis, particularly in elderly thin females. Gallbladder torsion requires operative management, and cannot be treated with a cholecystostomy, so pre-operative diagnosis is important.

\section{Acknowledgments}

None to declare.

\section{Financial Disclosure}

None to declare.

\section{Conflict of Interest}

None to declare.

\section{Informed Consent}

Informed consent was obtained prior to the completion of these reports.

\section{Author Contributions}

JC contributed towards writing of the manuscript. KD and AS provided advice, review and alterations to the manuscript prior to submission.

\section{Data Availability}

The authors declare that supporting data are available within this article.

\section{References}

1. Reilly DJ, Kalogeropoulos G, Thiruchelvam D. Torsion 
of the gallbladder: a systematic review. HPB (Oxford). 2012;14(10):669-672.

2. Wendel AV. VI. A case of floating gall-bladder and kidney complicated by cholelithiasis, with perforation of the gall-bladder. Ann Surg. 1898;27(2):199-202.

3. Copely K, Dawkins A. The floating gallbladder. Abdom Radiol (NY). 2020;45(10):3369-3370.

4. McHenry CR, Byrne MP. Gallbladder volvulus in the elderly. An emergent surgical disease. J Am Geriatr Soc. 1986;34(2):137-139.

5. Usui M, Matsuda S, Suzuki H, Ogura Y. Preoperative diagnosis of gallbladder torsion by magnetic resonance cholangiopancreatography. Scand J Gastroenterol. 2000;35(2):218-222.

6. Chou CT, Chen RC, Yang AD, Wu HK. Gallbladder torsion: preoperative diagnosis by MDCT. Abdom Imaging. 2007;32(5):657-659.

7. Alemi F, Seiser N, Ayloo S. Gallstone disease: cholecystitis, Mirizzi Syndrome, Bouveret Syndrome, gallstone ileus. Surg Clin North Am. 2019;99(2):231-244.

8. Treinen C, Lomelin D, Krause C, Goede M, Oleynikov D. Acute acalculous cholecystitis in the critically ill: risk factors and surgical strategies. Langenbecks Arch Surg. 2015;400(4):421-427.

9. Huffman JL, Schenker S. Acute acalculous cholecystitis: a review. Clin Gastroenterol Hepatol. 2010;8(1):15-22.

10. de Mestral C, Rotstein OD, Laupacis A, Hoch JS, Zagorski B, Alali AS, Nathens AB. Comparative opera- tive outcomes of early and delayed cholecystectomy for acute cholecystitis: a population-based propensity score analysis. Ann Surg. 2014;259(1):10-15.

11. Gurusamy K, Samraj K, Gluud C, Wilson E, Davidson BR. Meta-analysis of randomized controlled trials on the safety and effectiveness of early versus delayed laparoscopic cholecystectomy for acute cholecystitis. Br J Surg. 2010;97(2):141-150.

12. Gurusamy KS, Davidson C, Gluud C, Davidson BR. Early versus delayed laparoscopic cholecystectomy for people with acute cholecystitis. Cochrane Database Syst Rev. 2013;6:CD005440.

13. Balmadrid B. Recent advances in management of acalculous cholecystitis. F1000Res. 2018;7.

14. Kim SB, Gu MG, Kim KH, Kim TN. Long-term outcomes of acute acalculous cholecystitis treated by non-surgical management. Medicine (Baltimore). 2020;99(7):e19057.

15. Gardiner D, Yuide P. Acute gallbladder torsion: computed tomography and magnetic resonance imaging findings. ANZ J Surg. 2020.

16. Tajima Y, Tsuneoka N, Kuroki T, Kanematsu T. Clinical images. Gallbladder torsion showing a "whirl sign" on a multidetector computed tomography scan. Am J Surg. 2009;197(1):e9-10.

17. Dasyam AK, Koo J, Stahlfeld Miller M, Sell HW, Jr., Tublin ME. The cystic duct knot sign: case report with description of a new ultrasound sign of gallbladder torsion. Emerg Radiol. 2015;22(4):445-447. 